\title{
ENTRE EL CALCOLÍTICO Y LA EDAD DEL BRONCE Algunas consideraciones sobre la cronología campaniforme ${ }^{1}$
}

\author{
Patricia RÍOS \\ Concepción BLASCO \\ Raquel ALIAGA \\ Depto. de Prehistoria y Arqueología \\ $U A M$
}

\section{Resumen}

El trabajo aborda la problemática del Campaniforme como un fenómeno bisagra entre el Calcolítico y la Edad del Bronce, particularmente en el centro peninsular, donde las dataciones obtenidas confirman su implantación desde mediados del III milenio a. C. en convivencia con grupos Calcolíticos peninsulares y se prolonga, en su modalidad de estilo Ciempozuelos, hasta el final del primer cuarto del II milenio a. C. como un fenómeno paralelo al surgimiento de las primeras comunidades de la Edad del Bronce en la zona, lo que puede explicar ciertos paralelos tipológicos y sociales entre algunas manifestaciones del Bronce Antiguo y los últimos campaniformes.

Palabras clave: Calcolítico, Campaniforme, Edad del Bronce, cronología, carbono 14.

\section{Summary}

This paper raises the problem of Bell Beaker horizon as a phenomenon hinge between the Chalcolithic and Bronze Age. Particularly in the center of the Iberian Peninsula where dating obtained confirm its implementation since the midthird millennium, in coexistence with Chalcolithic groups. The presence of these Bell Beaker groups extends -with Ciempozuelos style, until the end of the first quarter of the second millennium, as a parallel phenomenon to the emergence of the first communities in the Bronze Age in this area. It could explain similarities between objects and social manifestations of the Bronze Age and the last Bell Beakers.

Keywords: Chalcolithic, Bell beaker. Bronze Age, chronology, 14C dating.

\section{INTRODUCCIÓN}

Como todas las divisiones de la Historia, las grandes etapas asignadas a la Prehistoria son sólo una forma de parcelar su larga trayectoria con el fin de hacer más tangible la evolución de las sociedades que la protagonizaron. Para estos tiempos más remotos de la Historia tales divisiones se fijaron atendiendo a una evolución tecnológica que, como cualquier otro tipo de rasgo, no

1 El trabajo ha sido financiado por los proyectos: "Patrimonio Arqueológico y Documental de la Comunidad de Madrid" (S2007/HUM 0543) Dirección General de Investigación de la Comunidad de Madrid. IP Concepción Blasco; y, "Las tiene una incidencia homogénea, ni temporal ni espacial en las distintas áreas y círculos. Por ello es frecuente que el factor tecno-industrial se vea sustituido o complementado por marcadores temporales que permiten definir límites más precisos. No obstante, a pesar de las mejoras que se han producido en los métodos de datación radiométrica, existe una dificultad real para medir los tiempos prehistóricos con cierta exactitud y resulta imposible llegar a datar un acontecimiento con-

sociedades calcolíticas y su marco temporal. Una revisión a la luz de nuevos datos (HAR2011-28731)". Ministerio de Ciencia y Tecnología. I.P. Corina Liesau. 
creto, por lo que siempre nos movemos con escasa precisión a la hora de aplicar estas divisiones a un territorio o a un determinado ámbito cultural.

En el caso que nos ocupa, el tránsito del Calcolítico a la Edad del Bronce en la Península Ibérica se ha fijado en torno al 2000 a. C., al asignar a la primera de estas etapas todo el III milenio a.C. mientras que la segunda se ha hecho coincidir con el II milenio a.C. Pero esta asignación nada tiene que ver con el cambio tecnológico que supuestamente diferenciaría estas dos fases o períodos ya que la novedad que marca ese tránsito, la adopción de una metalurgia basada en la aleación binaria de cobre y estaño, se incorpora en la inmensa mayoría de los círculos del Bronce peninsular a partir de mediados del II milenio, incluso entre los grupos más representativos y de mayor actividad metalúrgica como es el Argar.

Ello nos lleva a encasillar en la Edad del Bronce a una serie de grupos que en la mayor parte de su trayectoria son tecnológicamente calcolíticos, si entendemos como tales aquellos que practican una metalurgia basada exclusivamente en el cobre primario, aun cuando en las etapas más avanzadas llegan a conocer y dominar la metalurgia del bronce, de manera que su adscripción se realiza básicamente en función de su cronología y no de la tecnología que los define nominalmente. Pero tampoco esta identificación temporal resulta precisa si tenemos en cuenta las dataciones radiométricas calibradas obtenidas en los últimos años, ya que algunos de los yacimientos pertenecientes a estos grupos peninsulares considerados de la Edad del Bronce se encuentran perfectamente establecidos todavía dentro de los límites del III milenio.

Sin embargo, si tenemos en cuenta otros factores, se comprueba cómo estos grupos asignados a la Edad del Bronce pero con tecnología calcolítica presentan desde sus inicios características urbanísticas, sociales e incluso algunos rasgos tecnológicos y tipológicos novedosos que se alejan de los estándares calcolíticos. A esto se suma en muchos casos el abandono de sitios de prolongada ocupación calcolítica y la creación de poblados de nueva planta con características urbanística diferenciadas, por lo que desde un punto de vista sociocultural, estos cambios justifican su adscripción a una nueva etapa.
En contraposición, el fenómeno campaniforme se identifica como calcolítico, una asignación que quedaría justificada por la práctica de una metalurgia del cobre, pero también por su asociación, tanto espacial como temporal a los grupos calcolíticos a partir de mediados del III milenio a. C., cuyos asentamientos son coincidentes y además por el uso, en muchas ocasiones, de viejos monumentos megalíticos de enterramientos colectivos. Sin embargo su cronología desborda los límites del III milenio a. C. para prolongarse a lo largo del primer cuarto del II milenio a. C. de manera que, atendiendo a los valores numéricos de las dataciones que han proporcionado y a determinados rasgos socioeconómicos, nada impide el considerarlos tanto calcolíticos como de la Edad del Bronce, por lo que esta asociación calcolítico/campaniforme no resulta demasiado exacta. Prueba de ello es la similitud de la tecnología y tipología de determinadas piezas metálicas con los equipos de algunos grupos de la Edad del Bronce, así como algunos aspectos sociales, como es la aparición de los primeros indicios de sociedades jerarquizadas, o económicos, en especial los intercambios a larga distancia para la obtención de materias primas y objetos destinados a las élites.

Así pues, en general en la Prehistoria y en particular en esta etapa que nos ocupa más que en cualquier otro momento de la Historia, la fecha que marca este tránsito es solo una referencia y no un límite definido y rígido, como lo evidencia en el marco del tránsito del III al II milenio a. C., este caso paradigmático del Horizonte campaniforme, un fenómeno que sobrepasa el marco peninsular al alcanzar una extensión paneuropea con una larga trayectoria temporal que abarca en muchas regiones buena parte del III y II milenios a. C.

El panorama descrito es fiel reflejo de la multitud de factores que intervienen en el proceso de cambio de los diferentes grupos humanos y de la necesidad que los historiadores tenemos de analizar su peso en transformaciones sucesivas cambios que se van produciendo hasta entrar en una nueva era. Si ello es así, hay que tener en cuenta todos los factores que definen a un grupo y comprobar cómo, a lo largo de su trayectoria caracterizada por una serie de ítems que se mantienen más 
o menos constantes y que son los que lo identifican, existe una importante transformación de manera que las últimas generaciones muestran rasgos de una nueva etapa.

No resulta fácil mencionar todos y cada uno de los factores que permiten identificar a un determinado grupo social con una etapa concreta y mucho menos priorizarlos o determinar sus causas-efectos. Y lo que es más problemático, saber cuantificar la incidencia de cada uno de estos factores para determinar si responde a los rasgos de una determinada etapa o de otra, por ejemplo, que es más significativo ¿la cronología inicial o la cronología de las manifestaciones más tardías?; ¿su nivel tecnológico en relación con la metalurgia o su mayor o menor relación espacial con grupos calcolíticos o de la Edad del Bronce?; ¿su nivel de organización social?, ¿las características urbanísticas de sus asentamientos?, ¿determinadas manifestaciones funerarias?, etc. Demasiados problemas que resolver y en la mayoría de los casos poco tangibles y estrechamente relacionados entre sí, pero sin que tampoco resulte fácil establecer la secuencia de causa/efecto que existe entre ellos.

Con estas consideraciones sobre las dificultades existentes, el caso del Horizonte campaniforme, tal como lo entendemos, tiene todavía una problemática añadida y es la valoración de si estamos ante un fenómeno que afecta al conjunto de la sociedad del Calcolítico Final o estamos ante "unas modas", "unas pautas sociales", "una tecnología", "un sistema económico" que afecta sólo a un sector de la población de cierta relevancia -determinados grupos familiares, o sólo a unos individuos concretos-. En este caso nada impediría que estos grupos pudieran haber sido sincrónicos en un principio de sociedades calcolíticas y en un segundo momento de grupos de la Edad del Bronce, lo que facilitaría explicar una cronología coincidente con ambos. Pero el caso se complica si consideramos que, incluso en sus manifestaciones más tardías, en la mayoría de los casos se asocian a yacimientos netamente calcolíticos; mientras que la mayor parte de los grupos de la Edad del Bronce se instalan en lugares de nueva planta o se superponen a lugares previamente ocupados reordenando el espacio.
Ante tal situación, resulta indispensable un análisis detallado de las dataciones disponibles del Horizonte campaniforme peninsular que nos permitan ordenar los tiempos de toda su trayectoria y de sus características básicas para poder establecer comparaciones con algunos de los valores que han proporcionado tanto yacimientos Calcolíticos como de la Edad del Bronce.

\section{LA CRONOLOGÍA:}

En la actualidad contamos con un repertorio de dataciones relativamente amplio que nos han permitido fijar los límites del fenómeno campaniforme en el marco peninsular y contrastarlo con la cronología de este mismo horizonte en otros territorios europeos. Sin embargo, el importante número de dataciones todavía no es suficiente para conocer bien el desarrollo socioeconómico del horizonte, ya que la mayoría de las mediciones radiométricas se han realizado a partir de materiales aportados por contextos funerarios, mientras que los contextos residenciales, productivos y simbólicos no sólo no han entregado apenas dataciones, sino que son muy pocos los yacimientos documentados con estas funcionalidades y menos aun los que además tienen estratigrafías verticales bien definidas, pues la mayoría de ellos son campos de hoyos que presentan estratigrafías horizontales. A ello hay que sumar que estos contextos no funerarios apenas poseen elementos diagnósticos significativos para asignarlos a este horizonte.

Con todo, hoy estamos en condiciones de afirmar que el inicio del Campaniforme en la Península Ibérica está documentado en todas las regiones a partir de mediados del III milenio a. C. en fechas calibradas. Más problemático sigue siendo, en cambio, la inclusión de las fechas más antiguas manejadas desde hace años para la introducción del fenómeno campaniforme, así como definir sus últimas manifestaciones, presentes tanto en las fases finales de los grandes poblados calcolíticos, como en las primeras de los poblados del Bronce inicial. Sin embargo, no podemos dejar de valorar la serie de fechas antiguas que tradicionalmente han adelantado la llegada del campaniforme de estilo internacional en diversos puntos del centro de Portugal y el sur peninsular, en yacimientos clásicos como 
Zambujal, Porto Torrão o el Cerro de la Virgen de Orce, descartando como ya hicieran Castro y otros, las fechas de Terrera Ventura y Las Angosturas, procedentes de contextos poco claros (Castro et al., 1996: 106). No obstante las revisiones más recientes de las dataciones procedentes de yacimientos de estas regiones se aproximan al panorama general comentado no confirman dichas fechas antiguas (Valera, 2006).

Por ejemplo, en el sureste, las fechas de Los Millares llevan la aparición del campaniforme hacia el 2535 el 2400 a. C., al que seguirían al menos dos fases posteriores de las que no se tienen dataciones. En el caso de Los Castillejos las dataciones obtenidas son similares a las de Millares y muestran un intervalo que alcanza claramente el final del III milenio a. C. En el caso del Cerro de la Virgen, la aparición del campaniforme se sitúa en torno al 2465-2335 a C., mientras que los campaniformes incisos entrarían -desde el punto de vista temporal- en el denominado Bronce Antiguo alcanzando fechas del 2175- 1965 a. C. En definitiva en este área, atendiendo a las dataciones más recientes, tras un calcolítico precampaniforme anterior al 2450 a. C. un calcolítico campaniforme que abarca desde ese límite hasta el 2000/1750 a. C., el horizonte claramente argárico se instaura a partir del 1900 a. C (Molina et al., 2005).

Las dataciones conocidas para el suroeste son semejantes, según se desprende de la dinámica de algunos grandes poblados calcolíticos de fosos donde los contextos campaniformes se documentan de mediados del III milenio a inicios del II milenio a. C. Es, por ejemplo, el caso de La Pijotilla (4130 $\pm 40 \mathrm{BP})$, donde se evidencia la aparición del campaniforme a mediados del III milenio a. C. según se desprende de las fechas de la denominada tumba 2. Un momento en el que también se situaría el abandono del foso más externo y la construcción del foso semicircular (Márquez y Jiménez, 2010, 60-61), En algunos poblados calcolíticos de la Alta Andalucía, como Marroquíes, los campaniformes incisos, a juzgar por los cambios de estructuras sucedidos en el poblado y las manifestaciones funerarias, se consideran reflejo de transformaciones socioecnómicas propias de los grupos de la Edad del Bronce, sin embargo en la secuencia de este yacimiento la aparición del campaniforme se sitúa en la fase 3, ZAMB-3, con una cronología de mediados del III milenio a. C., 2450-2125 a. C. (Sánchez et al., 2005).

En este mismo sentido se ha interpretado que en algunos de los poblados del sur la presencia del campaniforme se asocia con un momento de desarticulación de los grandes asentamientos de tipo millarense, los cuales son sustituidos por aldeas aglomeradas de cabañas exentas frente a los aglutinados/centralizados previos identificados como aldeas-recinto. La causa de este cambio en los patrones de asentamientos se ha achacado a la desintegración social parental propia de la sociedad calcolítica en favor de un nuevo orden que procura la jerarquización de la sociedad y marca en la zona el arranque de la Edad del Bronce (Ramos Millán, 2004).

También en Portugal el campaniforme comienza a confirmarse como un fenómeno tardío, sobre todo en la zona la Extremadura portuguesa, donde, para algunos, se asienta en "las ruinas del antiguo modelo socioeconómico que generó los poblados fortificados", descartando así fechas antiguas como las de Leceia (Gonçalves, 2003: 312), de forma que el modelo de periodización del campaniforme en Portugal asumido actualmente dista de los primeros enunciados a mediados de los años 70 que basaron en datos escasos y dispersos. Hoy el panorama se ha enriquecido considerablemente tanto en número de fechas como en contextos fiables, y muestra un campaniforme que aparece en la última fase de los poblados fortificados y de fosos calcolíticos de toda la región.

Por otra parte, los últimos datos también parecen confirmar un primer momento de irrupción del campaniforme caracterizado por la presencia de cerámicas de estilo Internacional, no sólo en los yacimientos clásicos como Vila Nova de Sao Pedro, Zambujal o Rotura, cuyas dataciones y estratigrafía podrían considerarse confusas/dudosas, sino en otros en los que estas cerámicas han aparecido aisladas en estratos horizontales bien definidos. Es el caso de Porto Carretas (Soares, 2003:104) y otros poblados de la zona norte de Beira Alta, en los que este primer campaniforme no se asocia a metal (Silva, 
1991). Las fechas que se manejan para estos primeros momentos, tanto en la zona del estuario de Tajo, donde tradicionalmente se estimaban más antiguas y primer lugar de irrupción (Harrison, 1977), como en el resto de Portugal, se pueden situar entre en torno al $4000 \mathrm{BP}$ (Soares, 2003), es decir alrededor del $2500 / 2400$.

La aparición de las cerámicas incisas (grupo Palmela), pese a ser habitual junto a ejemplares impresos, está claramente asociada a un segundo momento cercano al abandono de los poblados calcolíticos y al inicio de la dispersión del poblamiento, que es interpretado como la desarticulación del modelo socioeconómico del Calcolítico (Soares, 2003: 105). Un yacimiento en el que se documentó este horizonte inciso, tanto en tumbas como en un nivel de ocupación del poblado, es Malhadas, cuyas fechas son del 3800-3600 BP (Soares y Tavares da Silva, 1974-77, 1984). Y del mismo modo que ocurre en el resto de la Península, el Bronce regional, por ejemplo en el poblado de Catujal, aporta fechas del $3570 \pm 45$ BP (2028-1751 cal a. C.). Otros contextos funerarios confirman también este intervalo y atestiguan las relaciones entre la meseta y la zona central de Portugal a partir de los paralelos materiales y la diversidad funeraria (adornos de oro, puntas de Palmela, puñales de lengüeta, cobre arsenical, tumbas individuales y reocupación de tumbas colectivas). Es el caso de la mayor parte de los hipogeos de la necrópolis de Quinta do Anjo y el tholos de Praia das Maçãs datados en $3600 \pm 80 \mathrm{BP}$ y $3760 \pm 80 \mathrm{BP}$, y $3650 \pm 50 \mathrm{BP}$ el segundo (Soares, 2003: 109-110). Por otra parte, el panorama descrito, coincide con el proceso observado en el sureste del que hemos tratado más arriba.

La situación del noroeste peninsular respecto al periodo campaniforme se ha visto mejorada en los últimos años con un aumento cualitativo y cuantitativo de los datos, que han sido recientemente recogidos en una monografía sobre el tema en Galicia (Martínez y Salanova, 2011). Las fechas manejadas para los contextos en que aparecen cerámicas campaniformes en más de un centenar de yacimientos muestran los valores más antiguos en los contextos de las ofrendas funerarias en determinados megalitos como el
Dolmen de Dombate o el Túmulo de Cotogrande entre el 2800 y el 2500 a. C., asociadas a ejemplares de estilo Internacional. Otros contextos denominados ceremoniales como Os Remedio o Guidoiro también presentan fechas del 28002400 (Prieto, 2011: 350). Sin embargo, no debemos olvidar que en su mayor parte se trata de fechas realizadas sobre carbón.

Según las tendencias estilísticas estudiadas por P. Prieto, los ejemplares incisos de la zona una vez más se asocian a fechas más recientes a partir de 2400 a. C. hasta los primeros siglos del II milenio a. C, en convivencia con otros estilos impresos y en un momento considerado en la zona como Bronce Inicial (Prieto, 2011). En la mayor parte de los yacimientos gallegos donde aparece campaniforme lo hace mostrando mezcla de estilos desde mediados del III milenio a. C., una situación muy semejante a la documentada en el norte de Portugal e incluso en la Extremadura portuguesa (Cardoso, 2005), aunque en estas zonas portuguesas el campaniforme se vincula a los asentamientos y tumbas calcolíticas o a las reutilizaciones de monumentos megalíticos, llegando en sus últimos momentos a convivir con los primeros yacimientos de la Edad del Bronce, a partir del 2200-1800 a. C., que no presentan cerámica campaniforme. Las fechas del norte de Portugal procedentes de yacimientos como Buraco da Pala I, Castro de Palheiros o Mirandela se sitúan en un intervalo de 2800-2350 a. C. aprox. en contextos en los que hay mezcla de estilos.

Teniendo en cuenta todas las fechas existentes, los problemas estratigráficos y la variabilidad de tipos cerámicos asociados al campaniforme en Galicia, P. Prieto y L. Salanova en una valoración general del período, concluyen que el intervalo en el que se define el campaniforme en el noroeste podría abarcar desde el 2600 al 1600 a. C., teniendo en cuenta que ambos extremos del intervalo no pueden concretarse con seguridad por las confusas estratigrafías de la zona (Prieto y Salanova, 2011: 386). Queda claro entonces que las fechas más antiguas sí se pueden relacionar a los ejemplares internacionales, y que en las fechas más recientes valoradas es el estilo epicampaniforme regional el mayoritario. 
Quedaría por resolver la consideración del campaniforme dentro del Bronce gallego a partir de mediados del III milenio a. C. en contraposición al Neolítico, aún aceptando -como en otras regiones- una clara sincronía entre las fechas de los últimos campaniformes con los primeros poblados de la Edad de Bronce (2200-1800 a. C.).

Muy cuestionadas son las fechas conocidas para algunos yacimientos del Valle del Ebro, con dataciones como las obtenidas a partir de restos óseos humanos de la tumba de la Atalayuela: $4060 \pm 60 \mathrm{bp} ; 4110 \pm 60$ BP y $4120 \pm 70$ BP (Barandiarán, 1978, y Andrés y Barandiarán, $2004,101)$ equivalentes a 2599,2700 y $2734 \mathrm{cal}$ BC (Castro et al., 1996), las cuales se asocian a vasos de ambos estilos. Si bien esta presencia de cerámicas de estilos impresos, incluido el internacional en contextos de estratigrafía mal definida, dejan abierta la posibilidad a una utilización del monumento de manera acumulativa (Andrés y Barandiarán, 2004), esta misma evidencia fue tomada para argumentar la posibilidad de que los estilos incisos, y en concreto el Ciempozuelos, pudieran estar presentes desde los momentos iniciales del fenómeno campaniforme (Harrison 1988),

En el levante mediterráneo, teniendo en cuenta el descarte de la fecha de la Cova de les Cendres, Cova del Tossal de la Font y Cova Puntasa (Castro et al., 1996), contamos con la fecha del poblado calcolítico al aire libre del Arenal de la Costa, asociada a la fase HTC (horizonte campaniforme de transición) que sitúa la ocupación campaniforme del poblado en $3890 \pm 80$ BP: 2484-2283 cal a. C. (Bernabeu et al., 1993: 41), una fecha que encajaría con el límite inferior que se propone para la etapa y poco antes del inicio del Bronce antiguo regional en torno al 2200/2100 a. C. (Pedro y Martí, 2004).

Los datos conocidos sobre la presencia del campaniforme en la cornisa cantábrica son poco precisos dada la escasez de fechas conocidas. Aunque, tal y como venimos mostrando, la aparición de las manifestaciones asociadas al fenómeno campaniforme se sitúan también a partir de 2400 en fechas calibras en yacimientos como la cueva de Los Husos (Castro et al. 1996).

\section{UNA NUEVA VISIÓN DEL CAMPANIFORME EN EL INTERIOR PENINSULAR.}

En estos últimos años la novedad más sobresaliente en el panorama que define el horizonte campaniforme y su transición a la Edad del Bronce la han aportado los nuevos datos obtenidos en yacimientos del interior peninsular, gracias sobre todo a las fechas obtenidas en la Región de Madrid a partir de yacimientos de reciente excavación. Si hasta ahora en esta zona del centro peninsular hemos tenido bien documentado el campaniforme Ciempozuelos en el tránsito del III al II milenio a. C., hoy no sólo se han aumentado las fechas y contextos conocidos sino que además se ha incrementado el lapso temporal sobre todo por dataciones procedentes de conjuntos con campaniformes impresos.

De los contextos con ejemplares campaniformes de estilo Internacional o impreso geométrico, los menos numerosos, conocemos desde hace algunos años las dos fechas de la Fase III del Túmulo de la Sima, con un importante conjunto de recipientes campaniformes de estilo Marítimo, o la de Valverde de Íscar -más imprecisa- asociada a campaniforme puntillado geométrico, que retrasan la cronología del campaniforme de estos estilos hasta el 2460 a.C. o 2470 a. C. respectivamente (Rojo et al., 2005; Delibes, 1979) según fechas calibradas obtenidas a partir de muestras de colágeno. Incluso el valor que aporta una fecha de Cerro del Bú procedente de un contexto habitacional con campaniforme marítimo se adentran en la primera mitad del III milenio, si bien su margen de error de más de 100 años (de Alvaro y Pereira, 1990, 205) obligan a tomarla con cierta precaución. Con estas dataciones la idea tradicional de la existencia de un horizonte campaniforme más antiguo representado en tumbas con ajuares campaniformes de estilo Internacional o impreso geométrico que parecía apuntarse en territorios de la Meseta Norte, empieza a confirmarse. Consideramos de este modo que las fechas existentes para ambas Mesetas son indicio suficiente de ello a pesar de que el intervalo de calibración ocupa gran parte de la segunda mital del III milenio a. C. Sólo el citado caso del Túmulo de la Sima cuyas fechas calibradas, aceptables, muestran un intervalo de 200 años para esa 
tumba, desde el 2460 al 2200 cal a. C., por tanto con un límite inferior -2200 a. C.

El campaniforme Ciempozuelos característico del interior peninsular, podemos adscribirlo desde hace años a los últimos siglos del III milenio a. C. e incluso primeros del II milenio a. C. según las fechas de Fuente Olmedo en Valladolid (Martín y Delibes, 1989: 81), Almenara (Delibes y Guerra, 2004) o el Túmulo 1 de Aldeagordillo en Ávila (Fabián, 2006). Esta cronología reciente del fenómeno campaniforme inciso en el interior peninsular ha quedado confirmada por las dataciones procedentes de la necrópolis del Valle de las Higueras (Huecas, Toledo) también con ajuares tipo Ciempozuelos (Bueno et al., 2005), a las que se suman las últimas fechas obtenidas en la explotación salinera de Molino Sanchón II, en Zamora, adscritas también a campaniforme Ciempozuelos qua aportan valores calibrados del 2400-2200 cal a.C. (Abarquero et al, 2010, 136) aunque al tratarse de dataciones obtenidas a partir de carbón no podemos compararlas con las anteriores por el considerable envejecimiento de la fecha que supone el carbón frente al hueso, aunque claramente entrarían en el mismo intervalo.

En Madrid, la única fecha de contexto claramente campaniforme era, hasta hace poco la de El Ventorro, que además se trataba de un contexto formado por ejemplares de estilo impreso geométrico, entre ellos un crisol, y de carácter doméstico. Pese a tener un importante margen de error, el intervalo en fechas calibradas volvía a situar este contexto en fechas del 2502-2228 cal a. C. (3880 $\pm 90 \mathrm{BP})$, en un momento claramente anterior a las fechas que se manejaban para los contextos campaniformes Ciempozuelos aunque se trata de nuevo de una datación sobre carbón (Priego y Quero, 1992, 368-369).

Las nuevas dataciones que ha aportado nuestro equipo y que proceden de contextos muy buenos relacionados con el horizonte son únicamente 8 procedentes de varios enterramientos de Camino de las Yeseras y Humanejos (Figura 1). Estas fechas muestran un desarrollo claro del fenómeno analizado en la segunda mitad del III milenio, con tres contextos que superan en antigüedad claramente el 2200 a. C. No nos parece casual que precisamente las tres dataciones más antiguas se correspondan con los dos contextos en los que aparecen ejemplares cerámicos de estilo Internacional o Marítimo, pertenezcan a tumbas donde posiblemente hubo más de un momento de inhumación, lo que también podría explicar, como en otros muchos enterramientos peninsulares, la asociación de ambos estilos, sin embargo la convivencia de estilos, como se ha podido comprobar en Portugal, no niega la existencia de dos momentos, el más antiguo y probablemente de menor incidencia, el correspondiente al estilo Internacional.

Tanto en Madrid como en el resto de la península aunque aún son pocos los yacimientos que muestran una fase en la que sólo están presente las vasijas de estilo Internacional, lo que sí se comprueba, como ha señalado Lazarich para la región andaluza (2004) y podemos ver en las fechas madrileñas, es que el tipo Marítimo desaparece con anterioridad al resto.

Los hallazgos de los últimos años en la Región central han confirmado la existencia de verdaderas necrópolis campaniformes como las conocidas en el oeste y sur peninsular asociadas a grandes poblados estables calcolíticos. Necrópolis de hasta ocho tumbas, como es el caso de Huecas, o una decena en Humanejos y siete en Camino de las Yeseras, un número incluso mayor a las clásicas del sur y de Portugal. Estos cementerios recientemente conocidos se construyen y están en uso en fechas del campaniforme Ciempozuelos -finales del III milenio y principios del II milenio a. C.- y presentan tumbas más o menos agrupadas o alineadas junto al poblado, al menos en Humanejos y Yeseras. En nuestra opinión no se trata de tumbas colectivas aunque en la agrupación de las mismas y asociación de varios individuos (hasta 4 no simultáneos) en una misma tumba recuerde un cierto carácter colectivo, seguramente familiar, una forma de vincularse a sus ancestros.

La construcción de estas tumbas en fosas complejas, de tipo hipogeo o en cueva artificial, en cierto modo monumentales, con acceso a veces escalonado, recuerda a las necrópolis de cuevas artificiales portuguesas o las tumbas de falsa cúpula del sur peninsular, una especie de "megalitismo tardío" (Bueno et al., 2007-08) o "segunda tradición megalítica" (García Sanjuán, 2005: 155) desarrollado por los grupos campani- 
fomes desde mediados del III milenio a. C. a juzgar por las fechas de Camino de las Yeseras, Humanejos, Huecas o Aldeagordillo, y que estarían en consonancia con las fechas de Portugal y del sur peninsular (Soares, 2003; García y Hurtado, 2001; Gonçalves, 1994, 2003, 2005); Esta idea de "recuperar la tradición megalítica" en el interior encaja con la reutilización habitual por parte de los grupos campaniformes de los sepulcros de falsa cúpula, túmulos y megalitos tanto del interior y como del resto de la Península Ibérica.

Como se observa en Portugal o en el sureste, el final de estos grandes poblados madrileños parece haberse producido a la vez que desaparece el registro de los grupos campaniformes Ciempozuelos. Concretamente en los primeros siglos del II milenio a. C.: se abandonan los últimos fosos y los grandes poblados en favor de las pequeñas aldeas y las grandes y ricas tumbas campaniformes son sustituidas por sepulturas más simples en hoyo o en pequeñas covachas artificiales que siguen una vieja tradición de la zona y que apenas cuentan con ajuar o incluso carecen de él.

En resumen, las nuevas fechas obtenidas en las estructuras campaniformes de la Región de Madrid nos han permitido confirmar que los enterramientos con ajuares de campaniforme Ciempozuelos se sitúan en el tránsito del III al II milenio a. C., entre el 2200 y el 1740 a. C. en fechas calibradas, mientras que las tumbas con ejemplares internacionales e impresos geométricos tienden a situarse en torno al 2500 a.C. Estas fechas y estilos coinciden aparentemente con arquitecturas algo diferentes, en una clara intención de los personajes Ciempozuelos por las grandes estructuras hipogeicas ${ }^{2}$, que recuerdan la idea megalítica del monumento funerario mientras que el tratamiento de los difuntos es individual, propio de un momento en el que se producen indicios de jerarquización. Cambios sociales característicos de la Edad del Bronce cuyos primeros poblados son sincrónicos, no solo en áreas tradicionalmente investigadas, como el Argar, sino también en la propia Región central ya que

2 Por el momento no podemos definir un modelo para las tumbas de ejemplares impresos aunque los últimos datos conocemos fechas de Madrid y de ambas mesetas que sitúan estos nuevos establecimientos en un intervalo casi paralelo a los últimos campaniformes en torno a los últimos siglos de los poblados calcolíticos (FIGURA 1).

\section{LA CUESTIÓN DE LA RELACIÓN DE LOS GRUPOS CAMPANIFORMES CON LAS PRIME- RAS MANIFESTACIONES DE LA EDAD BRONCE}

Como hemos apuntado, una de las grandes aportaciones que se derivan de trabajos recientes es la comprobación de la coexistencia, en varios yacimientos calcolíticos de la Región de Madrid, de manifestaciones funerarias, domésticas y productivas tanto de grupos campaniformes como no campaniformes, una sincronía avalada por dataciones que han dado un mismo intervalo y que cubren desde mediados del III milenio hasta final del primer cuarto del II milenio a.C. Se trata de tumbas individuales o múltiples, en hoyo simple y sin ajuar y de estructuras domésticas tipo cabaña o de suelos y cubetas con indicios de actividades productivas.

Por el contrario, la relación de los campaniformes con las primeras generaciones pertenecientes a la Edad del Bronce no es tan evidente, pues ni hay una relación espacial tan estrecha ni las dataciones tienen un solapamiento tan claro a la luz de las calibraciones obtenidas en algunos yacimientos del área argárica (Lull et al, 2010) y "sus confines", pero también con las conocidas del ámbito de Mancha o en las cuencas del Duero y del Tajo.

El ejemplo mejor conocido, por el elevado número de registros que tenemos, es el del área argárica para la que la visión tradicional propuso una cronología que sitúa su desarrollo entre 2500 y 1400 AC (Gonzalez, 1994:17 y Castro et al. 1996,120-128) un lapso en el que se enmarcarían cinco períodos, los dos primeros, identificados con el Argar A, cubren el último cuarto del tercer milenio BP y coinciden con los grupos campaniformes con decoraciones impresas, mientras que los otros tres períodos, asociados al denominado Argar B, coincidentes con la prime- 
ra mitad del II milenio BP, son sincrónicos a los campaniformes incisos, como el Ciempozuelos y otros estilos regionales.

Sin embargo si se atiende a las características de los conjuntos funerarios ha habido un acuerdo casi total de que solo es posible diferenciar dos grandes períodos, "el primero de ellos comprende desde el inicio del II milenio hasta $c$.
1800 cal. ANE....y el segundo desde $c .1800$ cal. ANE hasta el final del grupo argárico" (Castro et al. 2001, 191), en este caso el primer período caracterizado por la alabarda, como arma emblemática de la panoplia coincide con la mayoría de los enterramientos campaniformes individuales o, a lo sumo, de dos o tres individuos, con presencia de cerámicas tanto impresas como inci-

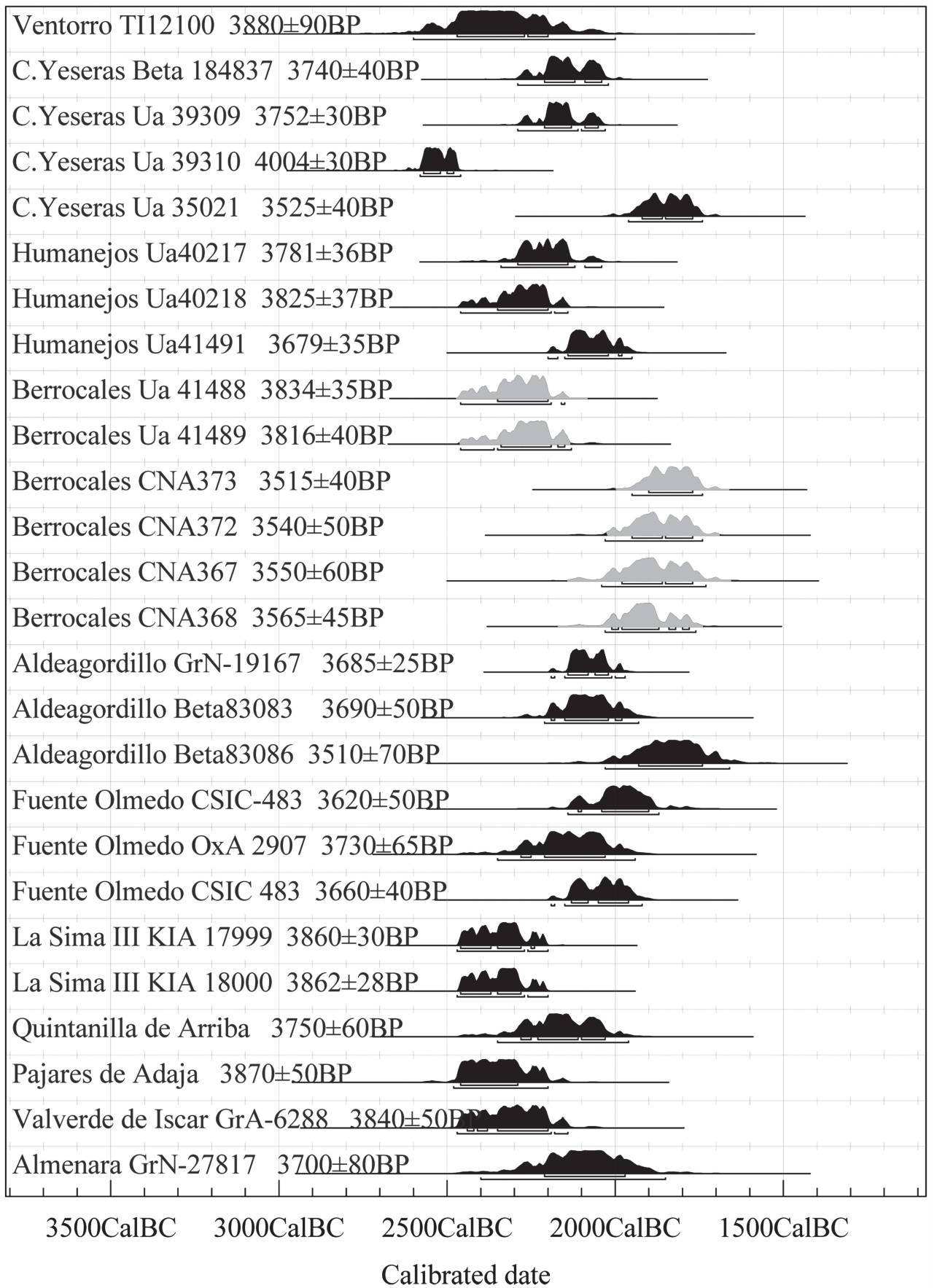

Campaniforme

Edad de Bronce

Figura 1: A-1 


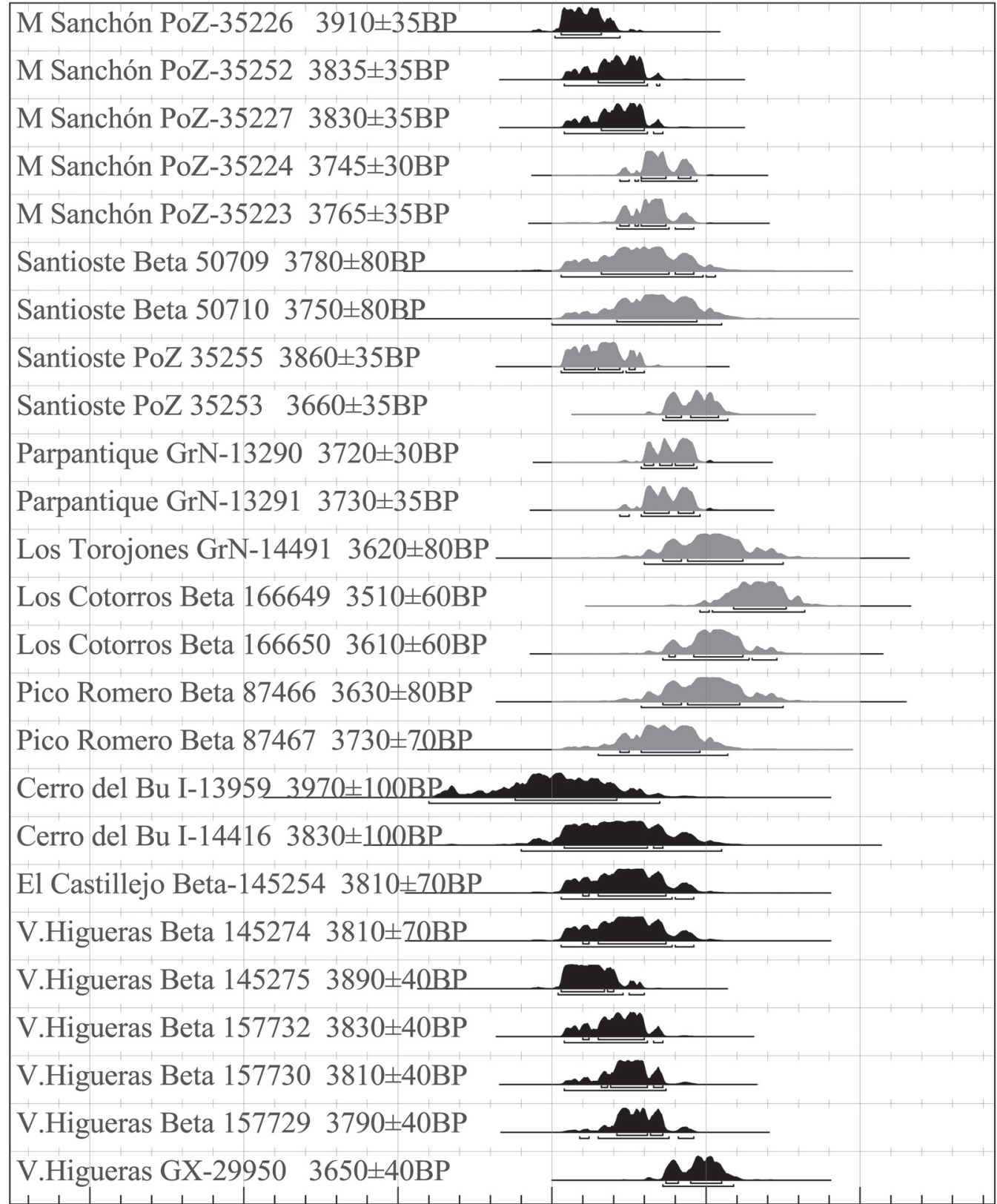

$4000 \mathrm{CalBC} 3500 \mathrm{CalBC} 3000 \mathrm{CalBC} 2500 \mathrm{CalBC} 2000 \mathrm{CalBC} 1500 \mathrm{CalBC}$

Calibrated date

Campaniforme

Edad de Bronce

Figura 2: A

sas, es decir sería sincrónico a la segunda etapa campaniforme. No obstante posteriormente esta secuencia argárica se ha rejuvenecido ligeramente simplificándola con una nueva periodización tripartita para la que proponen una cronología entre 2250/2200 y 1450/1400 a. C. La primera fase comprende hasta el 1900 haciéndola coincidir con el Bronce Antiguo, la segunda abarca entre 1900 y 1450 AC, identificándola con el Bronce Pleno, mientras que la tercera entre 1650 y $1450 \mathrm{AC}$, pertenece ya al Bronce Tardío (Molina y Cámara 2004, 456-457). En esta propuesta el Horizonte campaniforme sería contemporáneo de la primera etapa y buena parte de la segunda. 
Recientemente, autores que conocen bien el mundo argárico, teniendo en cuenta sólo dataciones sobre muestras de vida corta y estimaciones probabilísticas, así como la constatación de que en yacimientos como Gatas o casco urbano de Lorca los estratos calcolíticos subyacen a los niveles de la Edad del Bronce concluyen "que las comunidades calcolíticas se desarticularon antes de la instauración de las primeras manifestaciones materiales que reconocemos como argáricas. Sin embargo no hay que imaginar entre ambas un hiato o interludio prolongado" (Lull et al. 2010, 91), esta constatación explicaría la ausencia o, en todo caso, la anecdótica presencia del campaniforme en yacimientos argáricos y permitiría descartar una prolongada coetaneidad entre el Calcolítico y la Edad del Bronce del sureste, a la que se le había atribuido una mayor antigüedad que al resto de las regiones peninsulares.

En otros círculos de la Edad del Bronce con menor número de dataciones y sobre todo de mediciones tan precisas, solo algunas muestras puntuales han aportado valores que sobrepasan los límites del II milenio cal A.C, como es el caso de la Mancha tanto en la facies de las Motillas (Nájera y Molina, 2004, 40) como en la de los poblados de altura (Sánchez Meseguer y Galán, 2004, 160 y Sánchez Meseguer, 2004, 57) y por consiguiente también sus inicios coincidirían, solo de manera tangencial, con una parte de la secuencia temporal del Calcolítico, sobre todo teniendo en cuenta que en muchos casos se trata de muestras de vida larga o con una desviación muy alta por tanto este solapamiento se reduce sensiblemente. Esta coincidencia también la encontramos en círculos más septentrionales como el Bronce Valenciano o el turolense (Burillo y Picazo, 2001, 120) pero también, de manera esporádica ya que las mediciones que alcanzan cifras que sobrepasan el límite del 2000 A.C. son muy escasas. Por tanto, podemos deducir que de manera general Calcolítico-Edad del Bronce son estadios consecutivos con un límite que marca una cierta ruptura entre ellos.

Pero esta secuencia no resulta tan clara si nos referimos específicamente a los grupos campaniformes ya que las dataciones antiguas, pero sobre todo las nuevas series realizadas sobre muestras de vida corta y por AMS, son macha- conamente persistentes en aportar resultados que enmarcan este Horizonte en un amplio marco temporal que cubre desde mediados del III milenio cal A.C. hasta casi finales del primer cuarto del II milenio A.C., unos resultados que representan un escollo para adjudicar el campaniforme al Calcolítico de manera exclusiva, por lo que, al menos desde el punto de vista temporal, es evidente su identificación como Horizonte bisagra a caballo entre el calcolítico y la Edad del Bronce, el problema estriba en la ausencia o, a lo sumo, en la presencia testimonial que sus elementos identificadores $\mathrm{y}$, en particular la característica cerámica decorada, tienen en los yacimientos de la Edad del Bronce.

Con este panorama resulta complicado encajar todas las piezas, salvo que entremos a analizar la propia secuencia campaniforme y a considerar una trayectoria con dos etapas bien definida, la primera de ellas, caracterizada por la presencia de las cerámicas impresas, acompañadas o no de ejemplares incisos, cuyo marco temporal cubriría desde mediados a finales del III milenio A.C. y una segunda representada por los contextos de decoraciones exclusivamente incisas, como son la variedad Ciempozuelos y otros estilos regionales cuya cronología cubre el primer cuarto del II milenio aproximadamente. Desde esta perspectiva, resulta que la primera fase sería coetánea del Calcolítico mientras que la segunda etapa es coincidente con el Bronce Antiguo, aunque sigue sin encajar la escasa o nula presencia de ejemplares cerámicos campaniformes en los niveles iniciales de la Edad del Bronce.

Esta coincidencia temporal con Calcolítico o Edad del Bronce explica, entre otras cosas, algunos aspectos diferenciadores entre ambas fases del campaniforme, como son la mayor presencia de los ejemplares impresos en monumentos megalíticos levantados y utilizados previamente, o la incorporación de más cuerpos en una misma tumba, aun cuando a cada uno se les dé un cierto tratamiento individualizado, frente a los grupos tardíos con tendencia a ocupar tumbas individuales o en pareja, tal como es frecuente en la Edad del Bronce.

Sin embargo queda por resolver cómo son las relaciones entre los campaniformes y los grupos de la Edad del Bronce coetáneos. Una explicación podría ser que en aquellos círculos con 
mayor personalidad, donde la Edad del Bronce representa una ruptura bastante radical con respecto a las comunidades calcolíticas, el campaniforme regional prácticamente no existe, mientras que en aquellas áreas donde la Edad del Bronce es menos rupturista y su formación parece ser algo más tardía, el Campaniforme regional tiene una cierta pujanza, no obstante todo parece indicar que en estos casos, su presencia se mantiene sólo en los lugares ocupados por los grupos calcolíticos tardíos, quienes posiblemente, como en generaciones anteriores, aceptaban la autoridad de las élites campaniformes y únicamente, cuando esta autoridad no es reconocida, los lugares se abandonan y van surgiendo nuevos establecimientos con nuevos equipos renovados, aunque arquitectura y urbanismo no muestran cambios muy sustanciales y no sólo no hay presencia de enterramientos campaniformes más o menos singulares, sino que tampoco hay indicios tangibles de otro tipo de élites.

A pesar de ello algunos de estos lugares de la Edad del Bronce del interior debieron de crearse antes de la desaparición de las últimas jefaturas campaniformes, como lo avalan algunas dataciones puntuales del grupo Parpantique bien definido en el alto Duero o el grupo Loma del Lomo/Tejar del Sastre del entorno HenaresJarama-Manzanares. "En concreto [para el horizonte Parpantique] se dispone de una seriación de siete análisis válidos, cuyos resultados se encuadran en un margen superior al $90 \%$ de probabilidad, en el lapso 2350/1680, si bien se concentran entre 2010/1710 cal AC" (Fernández Moreno, 2010, 100). Pero también en el área del alto-medio Tajo donde a la luz de recientes dataciones realizadas por AMS sobre muestras de vida corta, (Figura 1), estos lugares de nueva planta debieron de haber perdido todo tipo de relación con los grupos calcolíticos y la autoridad campaniforme y su registro material.

En este escenario curiosamente se observa una mayor "contaminación" entre el grupo argárico y los campaniformes que entre éstos y las primeras generaciones de la Edad del Bronce del interior peninsular donde los grupos Ciempozuelos tienen una especial duración. Tales similitudes se advierten en ciertos comportamientos como son los símbolos que identifican a los líderes a través de panoplias de armas o de la incorporación en el ajuar funerario de determinados elementos con- feccionados con materiales costosos y exclusivistas, incluso en algunos casos ostentan idénticos objetos de prestigio: botones de marfil, "brazales de arquero" y más excepcionalmente alabardas, quizás porque es en estos dos ámbitos donde se mantiene la práctica de enterramientos singulares, mientras que entre otros grupos se asiste a la desaparición de tales inhumaciones, bien porque no hay una autoridad tan destacada, bien porque se pierde la práctica de una ritualidad funeraria singularizada en torno a las jefaturas.

\section{BIBLIOGRAFÍA}

ABARQUERO, F.J.; GUERRA, E.; DELIBES, G.; PALOMINO, A. L. y DEL VAL, J. (2010): Los yacimientos de Villafáfila (Zamora) en el marco de las explotaciones salineras de la Prehistoria europea. Junta de Castiila y León. Valladolid.

ALVARO, de E. y PEREIRA, J. (1990): "El Cerro del Bú (Toledo)". Actas del I Congreso de Arqueología de la Provincia de Toledo. Toledo, 199-213.

ANDRÉS, T. y BARANDIARAN, I., (2004): "La tumba calcolítica de la Atalayuela, treinta y cinco años después". Salduie, no 4, 85-124.

BARANDIARAN, I, (1978): "La Atalayuela: fosa de inhumación colectiva del Eneolítico en el Ebro Medio". Príncipe de Viana, 152-153, 381-422.

BERNABEU, J, FUMANAL, M.P., PASCUAL, J. L., PASCUAL, J, GUITART, I.; OROZCO, T.; BADAL, E.; BUXÓ, R.; VALLE, R. y CALVO, M. (1993): "El III milenio a. C. en el País Valenciano. Los poblados de Jovades (Concentaina) y Arenal de la Costa (Ontinyent)", Saguntum-PLAV, 26: 9-180.

BUENO, P., BARROSO, R. y BALBÍN, R. (2005): "Ritual campaniforme, ritual colectivo: La necrópolis de cuevas artificiales del Valle de las Higueras, Huecas, Toledo". Trabajos de Prehistoria, 62, $\mathrm{n}^{\circ}$ 2, 67-90.

BUENO, P., BARROSO, R. y BALBÍN, R. (2007-08): "Campaniforme en las construcciones hipogeas del megalitismo reciente al interior de la Península Ibérica". Veleia, 24-25, n² 2, 771-790.

BUENO, P., GILMAN. A., MARTÍN, C. y SÁNCHEZ-PALENCIA, F.J. (2010): Arqueología, sociedad, territorio y paisaje. Estudios sobre Prehistoria Reciente, Protohistoria y transición al mundo romano en Homenaje a $M^{a}$ Dolores Fernández Posse. Biblioteca Praehistorica Hispana, vol XXVIII. 
BURILLO, F. y PICAZO, J. (2001): “Prospección arqueológica y Edad del Bronce: una experiencia en la serranía turolense". En RUIZ-GÁLVEZPRIEGO, M. (coord.), 87-120.

CARdoso, J. L. (2007): Pre-história de Portugal. Ed. Verbo.

CASTRO, P.V., LULL, V. y MICO, P., (1996): Cronología de la Prehistoria Reciente de la Península Ibérica y Baleares (c. 2800-900 cal $A N E)$.. BAR International Series, 652. Oxford.

CASTRO, P.V., CHAPMAN, R., GILI, S., LULL, V., MICÓ, R., RIHUETE, C., RISH, C. y SANAHUJA, E. (2001): "La sociedad argárica”. En RUIZGÁLVEZ-PRIEGO, M. (coord.), 181-216.

DELIBES, G. (1979): “Hallazgos campaniforme en Villaverde de Iscar, Segovia: las variedades campaniformes contemporáneas de Ciempozuelos en la Meseta Norte", Boletín del Seminario de Estudios de Arte y Arqueología: BSAA, 45: 5-18.

DELIBES, G. y GUERRA, E. (2004): “Contexto y posible significado de un cuenco Ciempozuelos con decoración simbólica de ciervos hallado en Almenara de Adaja (Valladolid)", Zona arqueológica, $N^{o}$. 4, (Ejemplar dedicado a: Miscelánea en homenaje a Emiliano Aguirre. Arqueología): $116-125$

FABIÁN, J. F. (2006): El IV y III milenio en el Valle Amblés (Ávila). Monografías de Arqueología en Castilla y León, 5. Junta de Castilla y León.

FERNANDEZ MORENO, J.J. (2010): “Algunas consideraciones sobre la ocupación del territorio en los momentos iniciales de la Edad del Bronce en el Alto Duero”. En BUENO et al. (eds.), 95-114.

GARCÍA SANJUAN, L. (2005): “Las piedras de la memoria. La permanencia del megalitismo del Suroeste de la Península Ibérica en el II y I milenio a.n.e.", Trabajos de Prehistoria, 62 (2): 85109.

GARCÍA SANJUAN, L. Y HURTADO, V. (2001): “La arquitectura de las construcciones funerarias tipo tholos en el Suroeste de España. Investigaciones recientes", Aspetti del megalitismo prehistórico. Cagliari, 36-47.

GONÇALVES, V. (1994): “Sitios, 'horizontes' e artefactos 3. A questâo das grutas artificiais e os complexos funerarios de Alapraia e S. Pedro do Estoril no processo de calcolitizaçao do centro/sul de Portugal”, Arquivo de Cascais, 11: 31-94.

GONÇALVES, V. S. (2003): “Sítios, «Horizontes» e artefactos. Estudos sobre o $3 .^{\circ}$ milénio no Centro e Sul de Portugal", $2^{a}$ edição revista e aumentada com dois novos textos. Cascais: Câmara Municipal de Cascais.
GONÇALVES, V. (2005): Cascais hà 5000 anos. Cámara Municipal, Cascais.

GONZÁLEZ, P. (1994): "Cronología del grupo argárico”. Revista d'Arqueologia de Ponent, 4, 7-46.

HARRISON, R., (1977): The Bell Beaker cultures of Spain and Portugal. Peabody Museum. Harvard University.

HARRISON, R., (1988): "Bell Beakers in Spain and Portrugal: working whit radiocarbon dates in $\operatorname{tr} 3^{\text {rd }}$ milleniun BC", Antiquity, 62, nº 236, 464-472.

HURTADO, V. (2005): "El campaniforme en Extremadura. Valoración del proceso de cambio socioeconómico en las cuencas medias de Tajo y Guadiana", en Rojo et al. (coord): El campaniforme en la Península Ibérica y su contexto europeo. Universidad de Valladolid. Junta de Castilla y León: 321-349.

LAZARICH, M. (2004): "Balance actual de la investigación sobre el campaniforme en Andalucía occidental", III Simposio de Prehistoria Cueva de Nerja. Fundación Cueva de Nerja. Málaga: 393403

LULL; V., MICÓ, R., RIHUETE, C. y RISH, R. (2010): "Límites históricos y limitaciones del conocimiento arqueológico: La transición entre los gupos arqueológicos de Los Millares y El Argar”. En BUENO et al. (eds.), 75-94.

MÁRQUEZ, J. E. y JIMÉNEZ, V. (2010): Recintos de fosos. Genealogía y significado de una tradición en la Prehistoria del suroeste de la Península Ibérica (IV-III milenios A.C). Universidad de Málaga.

MARTÍN VALLS, R. y DELIBES, G. (1989): La cultura del Vaso campaniforme en las campiñas meridionales del Duero: el enterramiento de Fuente-Olmedo (Valladolid). Monografía del Museo Arqueológico de Valladolid, $2^{\mathrm{a}}$ edición.

MOLINA, F. y CAMARA, J.A. (2004): "La cultura del Argar en el área occidental del sureste". En HERNÁNDEZ, L. y HERNÁNDEZ, M.: La Edad del Bronce en tierras valencianas y zonas limítrofes. Villena-Alicante, 455-470.

MOLINA, F.; CÁMARA, J. A., CAPEL, J.; NÁJERA, T. Y SÁEZ, L. (2004): "Los Millares y la periodización de la Prehistoria Reciente del Sureste", Simposio de Prehistoria Cueva de Nerja. Fundación Cueva de Nerja: 142-158.

NÁJERA, J. y MOLINA, F. (2004): "Excavaciones en La Montilla del Azuer (Daimiel, Ciudad Real), 2000-2001", Investigaciones arqueológicas en Castilla La Mancha: 1996-2002: 35-48. 
PEDRO, M. J. y MARTÍ, B. (2004): “Los poblados de la cultura del Bronce valenciano”. En M. R. García y J. Morales (coord.): La península Ibérica en el II milenio a. C. Poblados y fortificaciones. Universidad de Castilla la Mancha, Cuenca: 299-333.

PRIEGO, C. y QUERO, S. (1992): El Ventorro, un poblado prehistórico de los albores de la metalurgia. Estudios de Prehistoria y Arqueología Madrileñas 8. Ayuntamiento de Madrid.

PRIETO, P. y SALANOVA, L. (2011) (coord): Las comunidades Campaniformes en Galicia. Cambios sociales en el III y II milenios BC en el $N W$ de la Península Ibérica. Diputación de Pontevedra.

PRIETO, P. (2011): "La alfarería de las comunidades campaniformes en Galicia: contextos, cronología y estilo", en Prieto y Salanova (coord.): Las comunidades Campaniformes en Galicia. Cambios sociales en el III y II milenios BC en el $N W$ de la Península Ibérica. Diputación de Pontevedra: 345-361.

RAMOS MILLÁN, A. (2004): "La evolución urbanística del asentamiento millarense. Un texto de historia social y política en la cultura tribal", III Simposio de Prehistoria Cueva de Nerja. Fundación Cueva de Nerja. Málaga: 404-424.

ROJO, M., GARRIDO, R. y MARTÍNEZ DE LAGRÁN, I. (2005) (coord.): El campaniforme en la Península Ibérica y su contexto europeo. Universidad de Valladolid. Junta de Castilla y León: 321-349.

SÁNCHEZ MESEGUER, J. L. (2004): "El yacimiento arqueológico del "Cerro de La Encantada",
Investigaciones arqueológicas en Castilla La Mancha: 1996-2002: 49-60.

SÁNCHEZ MESEGUER, J. L. y GALÁN, C. (2004): "El Cerro de la Encantada", en: Rosario García Huerta, Francisco Javier Morales Hervás (coord.): La Península Ibérica en el II milenio A. C.: poblados y fortificaciones: 115-172.

SÁNCHEZ, A., BELLÓN, J. P. y RUEDA, C. (2005): "Nuevos datos sobre la zona arqueológica de Marroquíes Bajos: el quinto foso", Trabajos de Prehistoria, 62 (2): 151-164.

SILVA, F. A. P. (1991): “Cerámicas campaniformes da bacia do Arda (Arouca-Oliveira de Azeméis) Aveiro: noticia preliminar". Actas de las IV Jornadas Arqueológicas (Lisboa 1990). Lisboa. Asociação dos Arqueólogos Portugueses: 237252.

SOARES, L. (2003): Os hipogeos da Quinta do Anjo (Palmela) e as economías do simbólico. Setúbal.

SOARES, L. y TAVARES DA SILVA, C. (1974/77): "O grupo de Palmela o quadro da cerámica campaniforme em Portugal”, O Arqueólogo Portugués, Serie III, Vols. VII-IX: 101-124.

SOARES, L. y TAVARES DA SILVA, C. (1984): “Le Groupe de palmela dans le cadre de a céramique campaniforme au Portugal", en Guilaine, J. (ed.): L'Age du Cuivre Euripéen. Civilisations a Vases Campaniformes, Toulouse: CNRS: 209-220.

VALERA, A. C. (2006): “A margen esquerda do Guadiana (região de Mourão), dos finais do $4^{\circ}$ aos inícios do $2^{\circ}$ milenio AC", Era-arqueologia, $n^{o}$ : $136-210$ 